05

\title{
Исследование спектров генерации арочных квантово-каскадных лазеров
}

\author{
() А.В. Бабичев ${ }^{1}$, Д.А. Пашнев ${ }^{2,3}$, Д.В. Денисов ${ }^{4}$, А.Г. Гладышев ${ }^{5}$, Ю.К. Бобрецова ${ }^{1}$, С.О. Слипченко ${ }^{1}$, \\ Л.Я. Карачинский ${ }^{1,5,6}$, И.И. Новиков ${ }^{1,5,6}$, Д.А. Фирсов ${ }^{2}$, Л.Е. Воробьев ${ }^{2}$, Н.А. Пихтин ${ }^{1}$, А.Ю. Егоров ${ }^{5}$ \\ ${ }^{1}$ ФТИ им. А.Ф. Иофффе РАН, \\ 194021 Санкт-Петербург, Россия \\ ${ }^{2}$ Санкт-Петербургский политехнический университет Петра Великого, \\ 195251 Санкт-Петербург, Россия \\ 3 Center for Physical Sciences and Technology, \\ LT-10257 Vilnius, Lithuania \\ ${ }^{4}$ Санкт-Петербургский государственный электротехнический университет „ЛЭТИ“ им. В.И. Ульянова (Ленина), \\ 197376 Санкт-Петербург, Россия \\ ${ }^{5}$ Университет ИТМО, \\ 197101 Санкт-Петербург, Россия \\ ${ }^{6} \mathrm{OOO}$ „Коннектор Оптикс“, \\ 197101 Санкт-Петербург, Россия \\ e-mail: a.babichev@mail.ioffe.ru
}

Поступила в редакцию 29.11.2019 г.

В окончательной редакции 15.01.2020 г.

Принята к публикации 18.02.2020 г.

\begin{abstract}
Представлены результаты исследования температурных характеристик арочных квантово-каскадных лазеров спектрального диапазона 7-8 $\mu \mathrm{m}$ с различными геометрическими размерами. Гетероструктура лазера была выращена методом молекулярно-пучковой эпитаксии. Активная область состояла из 50 каскадов на основе гетеропары твердых растворов $\operatorname{In}_{0.53} \mathrm{Ga}_{0.47} \mathrm{As} / \mathrm{Al}_{0.48} \mathrm{In}_{0.52} \mathrm{As}$. Продемонстрирована одночастотная генерации для лазеров с диаметром полукольцевого участка резонатора $100 \mu \mathrm{m}$ и длиной прямолинейной части в $1 \mathrm{~mm}$. Максимальный коэффициент подавления боковых мод при температуре $290 \mathrm{~K}$ составил $26 \mathrm{~dB}$, длина волны излучения $-7.73 \mu \mathrm{m}$.
\end{abstract}

Ключевые слова: квантово-каскадные лазеры, эпитаксия, фосфид индия, одночастотная генерация.

DOI: $10.21883 /$ OS.2020.06.49398.320-19

\section{Введение}

К настоящему моменту квантово-каскадные лазеры (ККЛ) используются в качестве компактных источников излучения средней инфракрасной области спектра. Особый интерес представляют одночастотные источники излучения. Формирование распределенной обратной связи (РОС) обеспечивает подавление продольных мод высшего порядка. В свою очередь, формирование решетки, обеспечивающей РОС на гетероструктуре [1-3], требует применения дорогостоящей электронной или голографической литографии. Использование же геометрии внешнего резонатора [4] наряду с большими геометрическими размерами также требует нанесения антиотражающего покрытия на переднее зеркало лазера и использование высокоотражающего покрытия на заднем зеркале лазера, что также приводит к существенному удорожанию конечного прибора.

Формирование лазерных резонаторов сложной формы позволяет исключить необходимость использования высокоточной литографии, а также внешних механических элементов. Результаты по формированию так называемых „,арочных“ ККЛ, состоящих из линейных и полукольцевых участков, представлены в работах [5-8]. Была достигнута лазерная генерация при комнатной температуре с коэффициентом подавления боковых мод на уровне $25 \mathrm{~dB}$ и пиковой выходной оптической мощностью на уровне $6 \mathrm{~mW}[8]$.

В настоящей работе представлены результаты по исследованию температурных характеристик ККЛ спектрального диапазона 7-8 $\mu \mathrm{m}$ с различными геометрическими размерами.

\section{Детали эксперимента}

Гетероструктура ККЛ была выращена компанией „Коннектор Оптикс“ на промышленной установке молекулярно-пучковой эпитаксии Riber 49, оснащенной твердотельным источником мышьяка, фосфора крекерного типа и источниками марки ABI 1000 для создания потоков галлия и индия $[9,10]$. В качестве подложки использовались пластины InP с ориентацией (001) и уровнем легирования $n=1 \cdot 10^{17} \mathrm{~cm}^{-3}$. В качестве буферного слоя был использован $\mathrm{In}_{0.53} \mathrm{Ga}_{0.47} \mathrm{As}$ (толщина составляет $500 \mathrm{~nm}, n=5 \cdot 10^{16} \mathrm{~cm}^{-3}$ ). Активная область включала 50 каскадов на основе гетеропары 
$\mathrm{In}_{0.53} \mathrm{Ga}_{0.47} \mathrm{As} / \mathrm{In}_{0.52} \mathrm{Al}_{0.48} \mathrm{As}$. Использована конструкция с двухфононной схемой опустошения нижнего уровня в каскаде $[11,12]$. Толщина верхней обкладки волновода (слоя InP) составила $3.9 \mu \mathrm{m}\left(n=1 \cdot 10^{17} \mathrm{~cm}^{-3}\right)$. В качестве контактных слоев использованы слои $\mathrm{In}_{0.53} \mathrm{Ga}_{0.47} \mathrm{As}$ толщиной 100 и $20 \mathrm{~nm}$ с уровнями легирования $1 \cdot 10^{17}$ и $1 \cdot 10^{19} \mathrm{~cm}^{-3}$ соответственно.

При формировании кристалла арочного ККЛ была применена конструкция мезы с затравом в подложку [13]. Ширина полоска вблизи поверхности гетероструктуры составила $16 \mu \mathrm{m}$. Исследованы два типа арочных резонаторов: с диаметром полукольцевого участка резонатора $100 \mu \mathrm{m}$ и длиной прямолинейной части резонатора $1 \mathrm{~mm}$, а также с диаметром полукольцевого участка резонатора $75 \mu \mathrm{m}$ и длиной прямолинейной части резонатора $3 \mathrm{~mm}$. Отражающее и антиотражающее покрытия на сколотые грани лазера не наносились. Монтаж кристалла на медный теплоотвод производился эпитаксиальной поверхностью вниз при помощи индиевого припоя.

Детектирование оптического сигнала производилось с помощью охлаждаемого фотоприемника $\mathrm{HgCdTe}$ (быстродействие порядка $100 \mathrm{~ns})$. Спектры стимулированного излучения измерялись с помощью фурье-спектрометра BrukerVertex 80v в диапазоне температур 77-300 K. Спектральное разрешение составляло $0.2 \mathrm{~cm}^{-1}$. Сигнал с фотодетектора оцифровывался внешним АЦП каждые $10 \mathrm{~ns}$ с запуском измерений по импульсам тока и усреднялся [14]. Спектр излучения записывался при достижении импульсом фотоответа максимального значения. Приведены графики спектров с максимальной интенсивностью. Вольт-амперные характеристики $(\mathrm{BAX})$ измерялись при пропускании импульсов тока с длительностью $70 \mathrm{~ns}$ и частотой следований $15 \mathrm{kHz}$.

\section{Результаты и их обсуждение}

BAX и ватт-амперные характеристики лазерных диодов с диаметром полукольцевого участка резонатора $100 \mu \mathrm{m}$ и длиной прямолинейной части резонатоpa $1 \mathrm{~mm}$ представлены на рис. 1,a. Пороговые напряжение $U_{\text {th }}$ и ток $I_{\text {th }}$ при температуре $77 \mathrm{~K}$ составляют $14.0 \mathrm{~V}$ и $1.95 \mathrm{~A}$ соответственно. С увеличением температуры наблюдается рост порогового тока. При температуре $300 \mathrm{~K}$ значения $U_{\text {th }}$ и $I_{\text {th }}$ составляют $14.4 \mathrm{~V}$ и $4.6 \mathrm{~A}$ соответственно. Аппроксимация экспериментальной зависимости $I_{\mathrm{th}}(T)$ с помощью формулы $I_{\mathrm{th}}(T)=B \exp \left(T / T_{0}\right)$, где $T_{0}$ - характеристическая температура стабильности порогового тока, $B$ константа, позволила определить значение $T_{0}$, которое находится на уровне $200-210 \mathrm{~K}$.

Спектры лазерной генерации при различном уровне токовой накачки, соответствующие температуре $77 \mathrm{~K}$, представлены на рис. 2, $a$. При пороговом значении тока $(I=2.0 \mathrm{~A})$ наблюдается генерация вблизи $1321 \mathrm{~cm}^{-1}$ $(7.57 \mu \mathrm{m})$. Спектр состоит из трех мод, отстоящих друг от друга на $1.3 \mathrm{~cm}^{-1}(\sim 7 \mathrm{~nm})$. Положение линий в спектре стимулированного излучения соответствует положению максимума интенсивности спектра спонтанного излучения лазера $\left(\sim 1320 \mathrm{~cm}^{-1}\right) \quad[9,14]$. Максимальный коэффициент подавления боковых мод (SMSR) находился на уровне $11 \mathrm{~dB}$. Полная ширина на полувысоте (FWHM) пика основной моды составила $1.4 \mathrm{~nm}$. С увеличением накачки наблюдается перераспределение интенсивностей боковых мод в спектре генерации. При токе $2.4 \mathrm{~A}$ возрастает интенсивность длинноволновой моды по отношению к основной моде с полным подавлением вклада коротковолновой моды (вблизи $1322 \mathrm{~cm}^{-1}$ ). Дальнейшее увеличение токовой накачки приводит к возникновению дополнительной моды вблизи $1314 \mathrm{~cm}^{-1}$ $(7.61 \mu \mathrm{m})$.

C повышением температуры происходит увеличение коэффициента подавления боковых мод при малом уровне накачки (вблизи порогового значения тока). При температуре $99 \mathrm{~K}$ максимальный коэффициент SMSR составил $16 \mathrm{~dB}$ (соответствует $I=1.9 \mathrm{~A}$ ). С увеличением уровня накачки возникают дополнительные моды в спектре генерации.

Дальнейшее увеличение температуры до $290 \mathrm{~K}$ (рис. $2, b)$ приводит к одночастотной генерации с максимальным коэффициентом подавления боковых мод $26 \mathrm{~dB}$ вблизи порогового значения тока $I=4.6$ А. Длина волны генерации соответствовала $7.73 \mu \mathrm{m}\left(1293 \mathrm{~cm}^{-1}\right)$. Полная ширина на полувысоте пика основной моды составила $2.0 \mathrm{~nm}$. С увеличением уровня накачки коэффициент подавления боковых мод падает. Одночастотная генерация с SMSR выше $16 \mathrm{~dB}$ наблюдается при значениях тока вплоть до $5.5 \mathrm{~A}$, что соответствует уровню накачки в $1.2 I_{\text {th }}$.

Проведем качественное описание селекции мод в арочном резонаторе. В силу наличия как линейной, так и полукольцевой частей в арочном резонаторе в спектре генерации данного составного резонатора могут присутствовать оптические моды составных частей резонатора, положение которых совпадает. На рис. 3 представлен спектр спонтанного излучения лазера [9]. Вертикальными линиями отмечены положения оптических мод в составных частях резонатора (синие линии соответствуют линейному резонатору, красные - полукольцевому резонатору). Представлен случай, когда положение основной оптической моды в полукольцевом резонаторе соответствует положению основной оптической моды в линейном резонаторе. Межмодовое расстояние $\Delta \lambda=\lambda^{2} / 2 n_{\mathrm{ef}} L$, где $n_{\mathrm{ef}}-$ эффективный показатель преломления, $L$ - длина резонатора, $\lambda-$ длина волны излучения, в линейном резонаторе составило $4.4 \mathrm{~nm}$ (для $n_{\mathrm{ef}}=3.28[15], L=1 \mathrm{~mm}$, синие линии на рис. 3 ). Величина $\Delta \lambda$ для мод шепчущей галереи в полукольцевом резонаторе диаметром $0.1 \mathrm{~mm}$ составила $55.8 \mathrm{~nm}$.

Как следует из рис. 3, $a$, положение второй оптической моды полукольцевого резонатора совпадает с положением 14-й моды линейной части резонатора, что приводит к значительной величине GMR (gain margin 

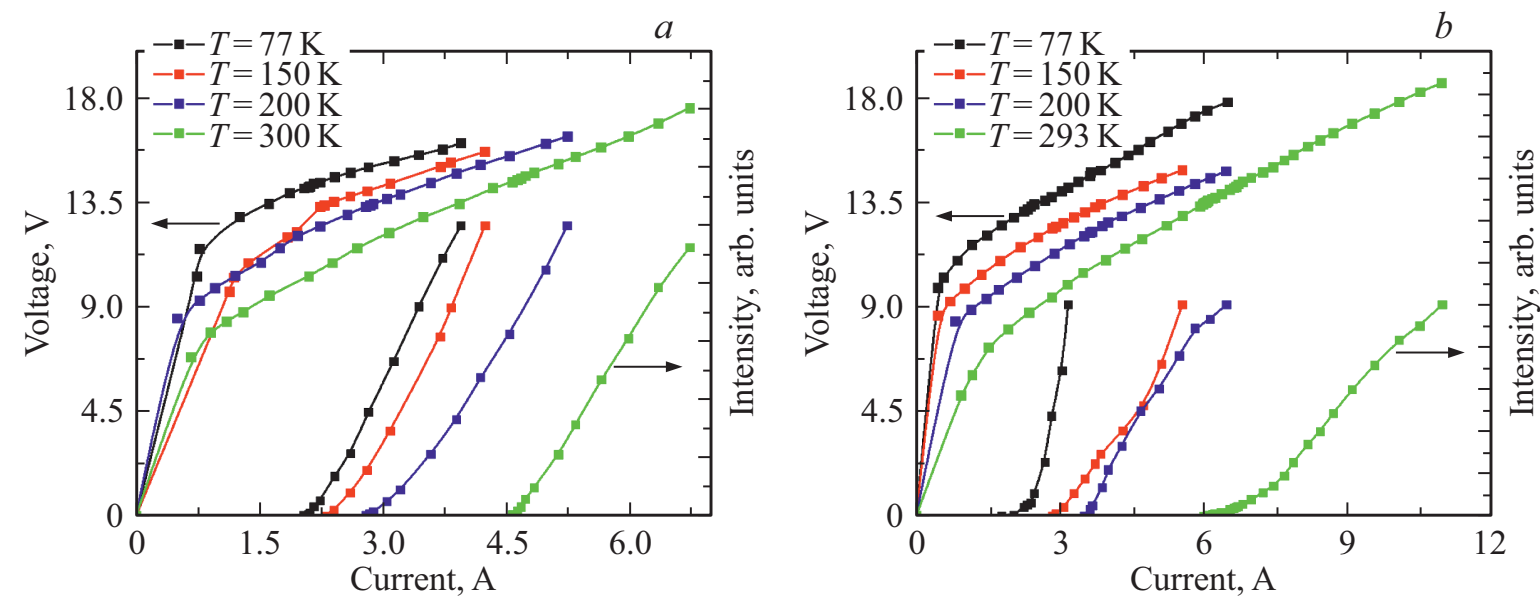

Рис. 1. ВАХ (левая ось ординат) и ватт-амперные (правая ось ординат) характеристики арочных ККЛ, измеренные при различных температурах. $a-\mathrm{c}$ диаметром полукольцевого участка резонатора $100 \mu \mathrm{m}$ и длиной прямолинейной части резонатора $1 \mathrm{~mm}$, $b$ - с диаметром полукольцевого участка резонатора $75 \mu \mathrm{m}$ и длиной прямолинейной части резонатора $3 \mathrm{~mm}$.

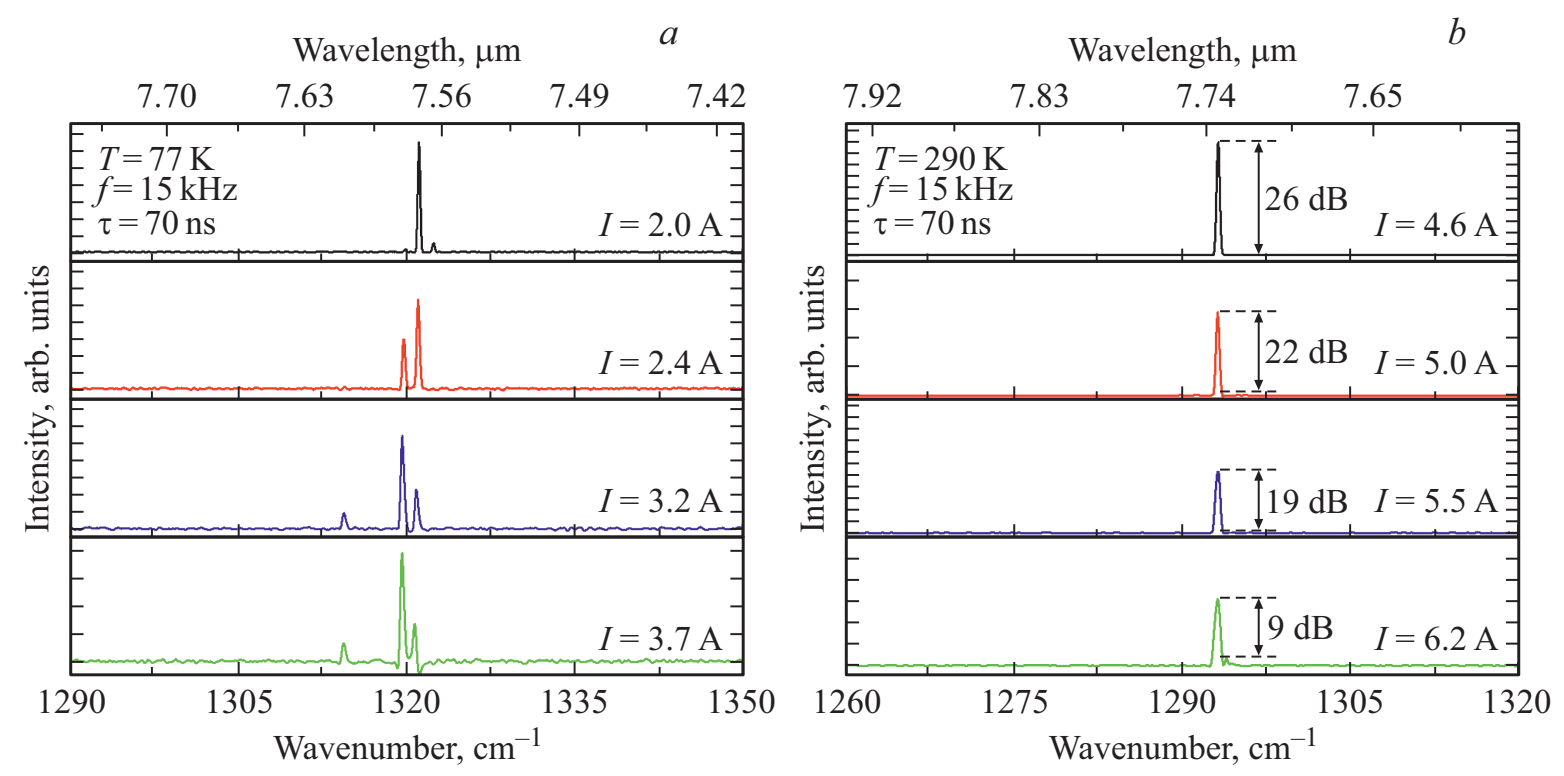

Рис. 2. Спектры генерации арочных ККЛ с диаметром полукольцевого участка резонатора $100 \mu$ т и длиной прямолинейной части резонатора $1 \mathrm{~mm} . a$ - при температуре $77 \mathrm{~K}, b$ - при температуре $290 \mathrm{~K}$.

ratio) в арочном резонаторе, характеризующей отношение между пиковым усилением и усилением, соответствующим положению ближайшей боковой моды, что может приводить к одночастотной генерации. В свою очередь, возможны и другие варианты расположения оптических мод составных частей арочного резонатора друг относительно друга, что может приводить к наличию нескольких мод в спектрах генерации (рис. 3,b). Не исключается также присутствие поперечных мод в спектрах генерации арочных ККЛ [6].

Наряду с исследованием арочных ККЛ с диаметром полукольцевого участка резонатора $100 \mu \mathrm{m}$ также проведены измерения характеристик арочных ККЛ с диаметром полукольцевого участка резонатора $75 \mu \mathrm{m}$. ВАХ и ватт-амперные характеристики арочных ККЛ с диамет- ром полукольцевого участка резонатора $75 \mu \mathrm{m}$ и длиной прямолинейной части резонатора $3 \mathrm{~mm}$ представлены на pис. $1, b$. Пороговые напряжение $U_{\text {th }}$ и ток $I_{\text {th }}$ при температуре $77 \mathrm{~K}$ составляют $12.5 \mathrm{~V}$ и $1.8 \mathrm{~A} \mathrm{соответствен-}$ но. С увеличением температуры до $293 \mathrm{~K}$ наблюдается рост пороговых напряжения и тока до $13.4 \mathrm{~V}$ и $6.0 \mathrm{~A}$ соответственно. Аппроксимация экспериментальной зависимости $I_{\mathrm{th}}(T)$ дает значения характеристической температуры стабильности порогового тока $T_{0}$ на уровне $190-210 \mathrm{~K}$.

Спектры генерации при различном уровне накачки представлены на рис. 4. При температуре $77 \mathrm{~K}$ продемонстрирована генерация вблизи $1340 \mathrm{~cm}^{-1}(7.46 \mu \mathrm{m})$, что соответствует коротковолновому плечу в спектре спонтанного излучения [14]. В спектре генера- 

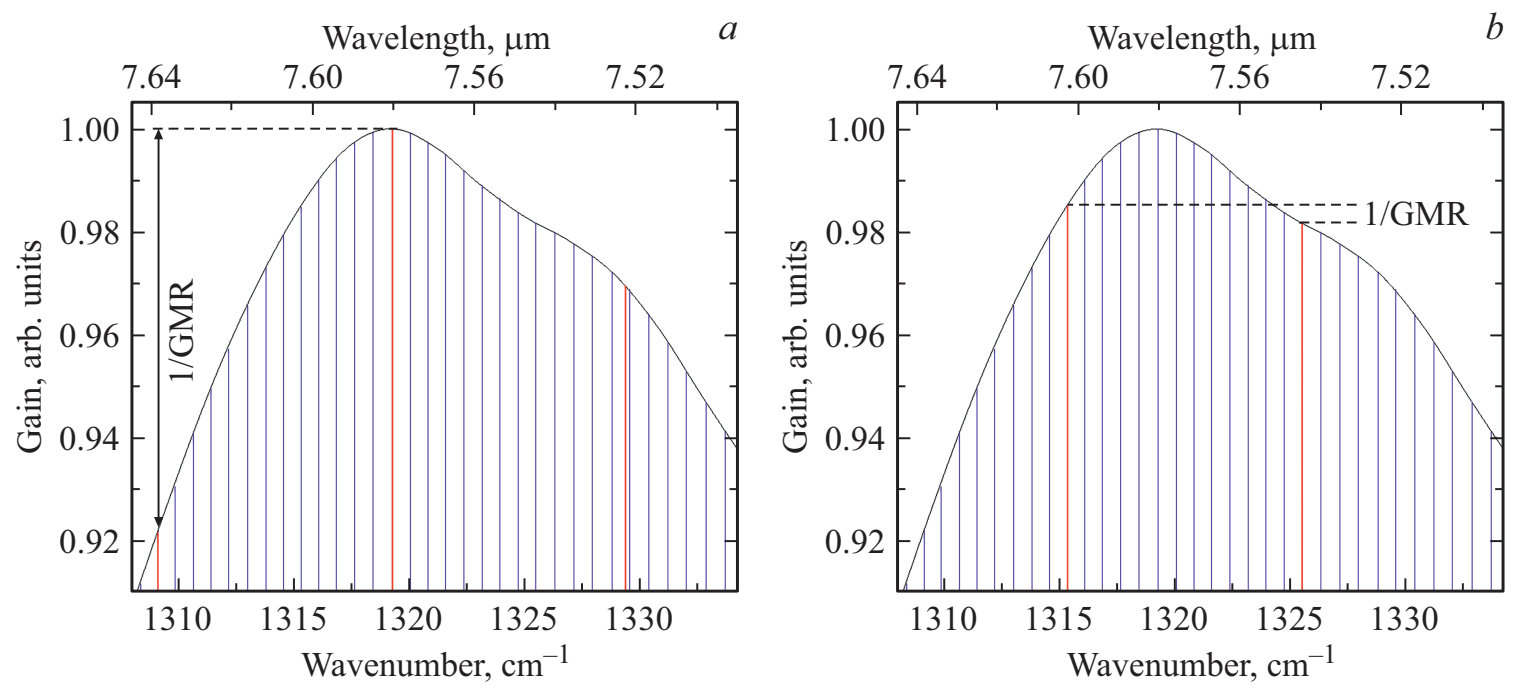

Рис. 3. Спектр спонтанного излучения лазера (нормированный на единицу). Синие линии соответствуют положению мод, соответствующих длине резонатора $2 \mathrm{~mm}$, красные - положению мод, соответствующих длине полукольцевого резонатора $(\pi \times 0.1 / 2) \mathrm{mm}$. $a-$ случай соответствия положения основной моды полукольцевого резонатора основной моде линейного резонатора; $b$ - случай, когда положение основной моды полукольцевого резонатора не соответствует положению основной моды линейного резонатора.
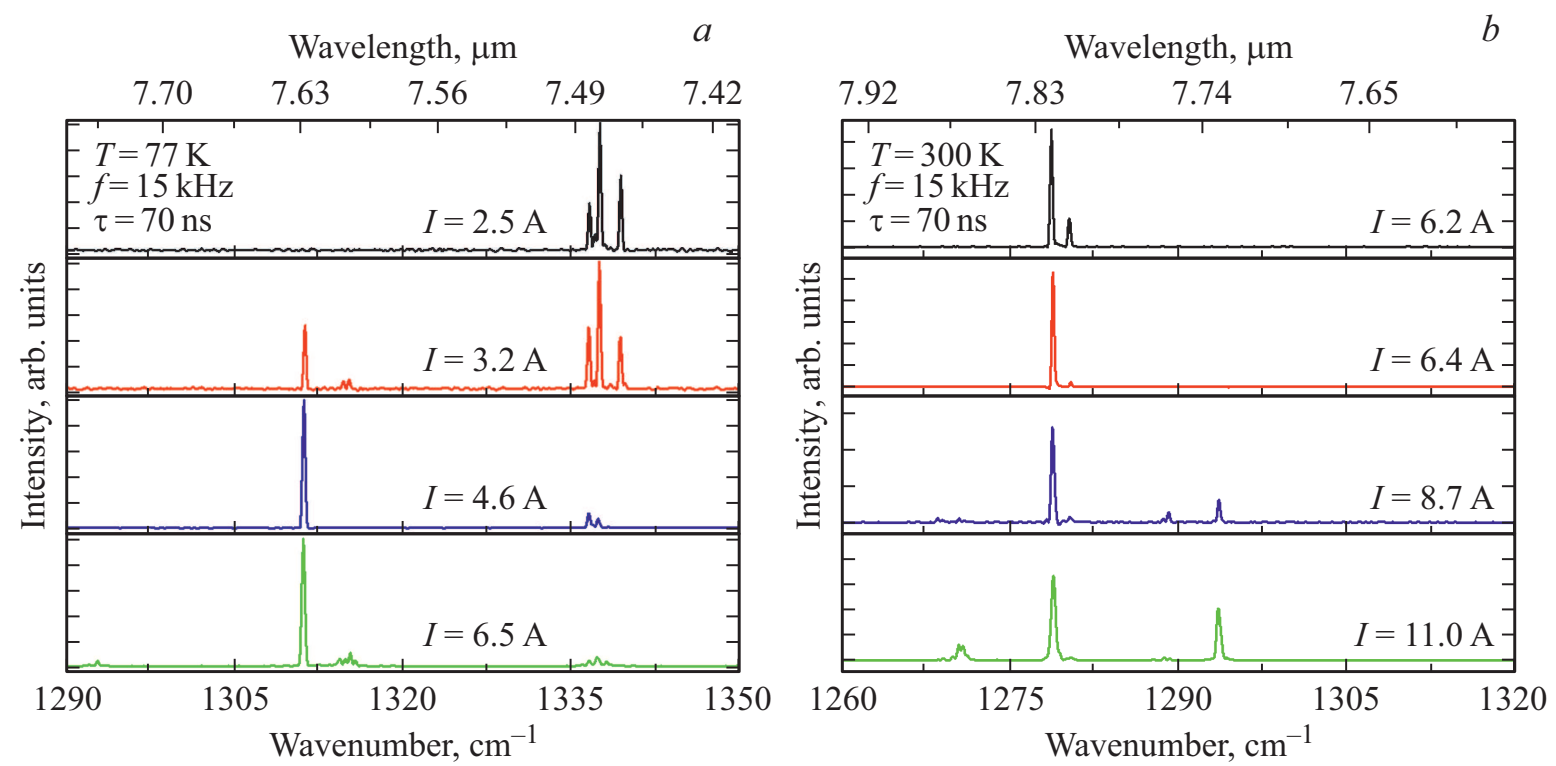

Рис. 4. Спектры генерации арочных ККЛ с диаметром полукольцевого участка резонатора $75 \mu \mathrm{m}$ и длиной прямолинейной части резонатора $3 \mathrm{~mm} . a$ - при температуре $77 \mathrm{~K}, b$ - при температуре $300 \mathrm{~K}$.

ции при уровне накачки $2.5 \mathrm{~A}$ наблюдаются три моды, отстоящие друг от друга на 0.97 и $1.81 \mathrm{~cm}^{-1}$ соответственно. Дальнейшее увеличение токовой накачки приводит к возникновению дополнительной группы мод вблизи $1311 \mathrm{~cm}^{-1}(I=3.2 \mathrm{~A})$, а также вблизи 1292 и $1315 \mathrm{~cm}^{-1}(I=6.5 \mathrm{~A})$.

В диапазоне температур $155-300 \mathrm{~K}$ в спектре генерации наблюдается несколько оптических мод. Коэффициент подавления боковых мод при малом уровне накачки находится на уровне 5-14 dB. Типичные спектры генерации, измеренные при температуре $300 \mathrm{~K}$, представлены на рис. 4, $b$. При малом уровне токовой накачки $(I=6.2 \mathrm{~A})$ в спектрах генерации наблюдается две моды, отстоящие друг относительно друга на $1.5 \mathrm{~cm}^{-1}(\sim 2.5 \mathrm{~nm})$. Максимальный коэффициент подавления боковых мод составил $14 \mathrm{~dB}$ (соответствует току накачки $6.4 \mathrm{~A}$ ). При токе свыше $8 \mathrm{~A}$ возникают дополнительные моды вблизи 1270, 1289 и $1294 \mathrm{~cm}^{-1}$. Ранее в работе [8] было показано, что при диаметре полукольцевого участка резонатора $75 \mu \mathrm{m}$ и длине прямолинейной части $1 \mathrm{~mm}$ лазерная генерация наблюдается вблизи $1293 \mathrm{~cm}^{-1}(7.73 \mu \mathrm{m})$. В свою очередь, с увеличе- 
нием длины прямолинейной части арочного резонатора с 1 до $3 \mathrm{~mm}$ продемонстрирована более длинноволновая генерация (вблизи $1279 \mathrm{~cm}^{-1}$ ) с подавлением составляющей вблизи $1293 \mathrm{~cm}^{-1}$ и коэффициентом подавления боковых мод порядка $14 \mathrm{~dB}$.

\section{Заключение}

Проведено исследование температурных характеристик арочных ККЛ спектрального диапазона 7-8 $\mu \mathrm{m}$. Продемонстрировано увеличение коэффициента подавления боковых мод с повышением температуры. Использование конструкции арочного резонатора с диаметром полукольцевой части 75-100 $\mu \mathrm{m}$ и длиной прямолинейной части $1 \mathrm{~mm}$ обеспечивает одночастотную генерацию при комнатной температуре с коэффициентом подавления боковых мод порядка $25 \mathrm{~dB}$. Одночастотная генерация при комнатной температуре соответствует длине волны излучения $7.73 \mu \mathrm{m}$. Полная ширина на полувысоте спектра лазерной генерации составила $2 \mathrm{~nm}$. Произведена оценка характеристической температуры стабильности порогового тока $T_{0}$ в арочных ККЛ, которая находится в диапазоне $190-210 \mathrm{~K}$.

\section{Финансирование работы}

Работа выполнена при частичной финансовой поддержке Российского фонда фундаментальных исследований в рамках научного проекта № 16-29-03289. Д.А. Фирсов, Л.Е. Воробьев благодарят за частичную поддержку исследований Минобрнауки России (государственное задание № 3.933.2017/4.6).

\section{Конфликт интересов}

Авторы заявляют, что у них нет конфликта интересов.

\section{Список литературы}

[1] Wu D.H., Razeghi M. // APLMater. 2017. V. 5. P. 035505. doi 10.1063/1.4978810

[2] Shahmohammadi M., Kapsalidis F., Süess M.J., Gini E., Beck M., Hundt M., Hundt M., Tuzson B., Emmenegger L., Faist J. // Semicond. Sci. Technol. 2019. V. 34. P. 083001. doi 10.1088/1361-6641/ab2838

[3] Hinkov B., Hayden J., Szedlak R., Martin-Mateos P., Jerez B., Acedo P., Strasser G., Lendl B. // Opt. Express. 2019. V. 27. P. 14716. doi 10.1364/oe.27.014716

[4] Maithani S., Maity A., Pradhan M. // Chem. Phys. 2019. V. 522. P. 123-128. doi 10.1016/j.chemphys.2019.02.022

[5] Liu P.Q., Sladek K., Wang X., Fan J.-Y., Gmachl C.F. // Appl. Phys. Lett. 2011. V. 99. P. 241112. doi 10.1063/1.3664117

[6] Liu P.Q., Wang X., Fan J.-Y., Gmachl C.F. // Appl. Phys. Lett. 2011. V. 98. P. 061110. doi 10.1063/1.3554757

[7] Zheng M.C., Zhang Y.M., Liu P.Q., Wang X., Fan J.-Y., Troccoli M., Gmachl C.F. // Opt. Eng. 2017. V. 57. P. 011001. doi 10.1117/1.oe.57.1.011001
[8] Babichev A.V., Gladyshev A.G., Kurochkin A.S., Dudelev V.V., Kolodeznyi E.S., Sokolovskii G.S., Bugrov V.E., Karachinsky L.Ya., Novikov I.I., Denisov D.V., Ionov A.S., Slipchenko S.O., Lyutetskii A.V., Pikhtin N.A., Egorov A.Y. // Tech. Phys. Lett. 2019. V. 45. P. 39. doi 10.1134/s1063785019040205

[9] Babichev A.V., Gusev G.A., Sofronov A.N., Firsov D.A., Vorob'ev L.E., Usikova A.A., Zadiranov Yu.M., Ilinskaya N.D., Nevedomskii V.N., Dyudelev V.V., Sokolovskii G.S., Gladyshev A.G., Karachinsky L.Ya., Novikov I.I., Egorov A.Y. // Tech. Phys. 2018. V. 63. P. 1511. doi 10.1134/s1063784218100043

[10] Babichev A.V., Kurochkin A.S., Kolodeznyi E.C., Filimonov A.V., Usikova A.A., Nevedomsky V.N., Gladyshev A.G., Karachinsky L.Ya., Novikov I.I., Egorov A.Y. // Semiconductors. 2018. V. 52. P. 745. doi 10.1134/s1063782618060039

[11] Babichev A.V., Gladyshev A.G., Filimonov A.V., Nevedomskii V.N., Kurochin A.S., Kolodeznyi E.S., Sokolovskii G.S., Bugrov V.E., Karachinsky L.Ya., Novikov I.I., Bousseksou A., Egorov A.Y. // Tech. Phys. Lett. 2017. V. 43. P. 666. doi 10.1134/s1063785017070173

[12] Babichev A.V., Gladyshev A.G., Kurochkin A.S., Kolodeznyi E.S., Sokolovskii G.S., Bougrov V.E., Karachinsky L.Ya., Novikov I.I., Bousseksou A., Egorov A.Y. // Semiconductors. 2018. V. 52. P. 1082. doi 10.1134/s1063782618080031

[13] Babichev A.V., Bousseksou A., Pikhtin N.A., Tarasov I.S., Nikitina E.V., Sofronov A.N., Firsov D.A., Vorobjev L.E., Novikov I.I., Karachinsky L.Ya., Egorov A.Y. // Semiconductors. 2016. V. 50. P. 1299-1303. doi 10.1134/s1063782616100067

[14] Babichev A.V., Pashnev D.A., Gladyshev A.G., Kurochkin A.S., Kolodeznyi E.S., Karachinsky L.Ya., Novikov I.I., Denisov D.V., Boulley L., Firsov D.A., Vorobjev L.E., Pikhtin N.A., Bousseksou A., Egorov A.Yu. // Tech. Phys. Lett. 2019. V. 45. P. 1136. doi 10.1134/S106378501911018X

[15] Babichev A.V., Dudelev V.V., Gladyshev A.G., Mikhailov D.A., Kurochkin A.S., Kolodeznyi E.S., Bougrov V.E., Nevedomskiy V.N., Karachinsky L.Ya., Novikov I.I., Denisov D.V., Ionov A.S., Slipchenko S.O., Lutetskiy A.V., Pikhtin N.A., Sokolovskii G.S., Egorov A.Yu. // Tech. Phys. Lett. 2019. V. 45. P. 735. doi 10.1134/s1063785019070174 